\title{
Editorial
}

Neuro[mmunoModulation

Published online: November 26, 2008

\section{An Introduction to Neuroimmunomodulation and Aging}

\author{
Moisés Evandro Bauer ${ }^{a} \quad$ Mónica De la Fuente ${ }^{b}$ \\ ${ }^{a}$ Faculdade de Biociências and Instituto de Pesquisas Biomédicas, Pontifícia Universidade Católica do Rio Grande \\ do Sul (PUCRS), Porto Alegre, Brazil; ' ${ }^{b}$ Departamento de Fisiología (Fisiología Animal II), Facultad de Ciencias \\ Biológicas, Universidad Complutense, Madrid, Spain
}

Aging is associated with gradual remodeling changes in various if not all systems in our body. During the last 50 years, several theories have been put forward to explain aging, including the free radical, neuroendocrine and immunological theories. Recently, accumulating evidence suggests that changes in the communication between major allostatic systems, including the immune and nervous systems (and concomitant loss of homeostasis and resistance to stress), is one of the fundamental causes of physiological senescence.

However, it is difficult to find out whether with age neural changes induce immunological changes or whether an impaired immune system induces nervous changes, or whether both processes occur simultaneously, which is the most likely mechanism according to some authors. The clinical consequences of the impaired communication between the immune and neuroendocrine systems may include increased susceptibility to infectious diseases, neoplasias and autoimmune disease. However, this altered morbidity is not evenly distributed and might be influenced by external or epigenetic factors, including psychological stress. Indeed, recent evidence suggests that psychological distress and stress factors are not only involved in immunosenescence but can also be associated with premature aging. Therefore, interventions aimed to ameliorate the changes in the neuroimmune communication during aging would be of special interest for the (c) 2008 S. Karger AG, Basel

1021-7401/08/0156-0211\$24.50/0

Fax +41613061234

E-Mail karger@karger.ch

www.karger.com
Accessible online at:

www.karger.com/nim gerontologists. Accordingly, this special issue of Neuroimmunomodulation provides an opportunity to review progress in this field of research.

An introductory article presented by De la Fuente reviews the most widely accepted theories of aging, especially the oxidation-inflammation theory, and how the neuroimmune communication is impaired with age. Moreover, she proposes that dysregulation of the immune system, in the context of the neuroimmune network, may contribute to the chronic oxidative stress that underlies aging.

Additional work reviews the fundamental concepts of the immunosenescence field, including remodeling changes of the immune system and inflammaging (see Ostan et al.). This work also reviews the role of the polymorphism of several genes involved in the immune response and inflammation in centenarians. The centenarians are the best example of successful aging, since they have avoided major age-related pathologies and are equipped with a well-preserved and efficient immune system. Preliminary data suggest that centenarians have increased plasma stress hormones (CRH, ACTH and cortisol) but show similar monocyte migration towards a bacterial chemotactic product (f-MLP) or neuropeptides when compared to young adults (see Genedani et al.).

Two papers review the concept that chronic psychological stress may premature the aging of neuroendocrine 
and immune systems. In particular, Gouin et al. review the impact of chronic stress experienced by elderly caregivers on the immune system. The chronic stress-induced immune dysregulation observed among older caregivers appears to be of sufficient magnitude to impact health. Bauer reviews recent work suggesting that extremely healthy aging is also associated with significant distress and neuroimmunomodulation changes related to increased glucocorticoid exposure. Preliminary data, suggesting that strictly healthy elders are somewhat protected from chronic stress exposure (caregiving stress) and show normal neuroendocrine and immune functions, are also discussed.

One of the most evident age-related change in the neuroimmune communication is the impaired innervation of lymphoid organs, altering various immune functions. The effect of age on sympathetic neurotransmission in an immune organ such as the spleen is discussed in the first original research article of the present issue by Bellinger et al.

In addition, the neuroendocrine function correlates with the immune function and the lack of appropriate hormonal levels during aging could be another factor of immunosenescence. Two articles of this issue present the immunoregulatory roles of hormones such as melatonin and growth hormone during the aging process. For instance, Cardinali et al. review the role of melatonin enhancing the immune function in aged individuals. Baeza et al. studied the effects of growth hormone administration on improving key immune function parameters in old male rats. Leposavic and Perisic summarize recent data on age-related thymic involution and its relation with gonadal steroids either directly or indirectly through catecholamines. Moreover, the role of the intrathymic sex steroid/catecholamine production in this involution is also considered.
The review presented by Sparkman and Johnson discusses how the age-related increase in neuroinflammation sensitizes the aged brain to cause an exaggerated inflammatory response to a stressor, which has a profound effect on behavior and cognitive function.

The key role played by the neuroimmunoendocrine network in the etiopathogenesis of the most common age-related neurodegenerative disorder, Alzheimer's disease, is reviewed by Gimenez-Llort et al. Moreover, using male and female triple-transgenic $3 \times \mathrm{Tg}$ - $\mathrm{AD}$ mice, these authors show a premature immunosenescence in these animals as well as the relevance of gender differences.

The neuroimmunomodulation interventions capable to attenuate immunosenescence are reviewed in two articles. Mocchegiani and Malavolta suggest the use of some compounds as new antiaging strategies in old animals and humans. The review by Pfister and Savino discusses possible ways of immune- and/or hormone-based interventions to delay or reverse immunosenescence.

Finally, Faria et al. report the frequencies of major lymphocyte subpopulations in a large cohort of aging individuals in Brazil. The age-related changes were not progressive and equally steady for all cell populations tested, but instead they showed a rhythmic behavior at different age intervals.

In conclusion, we express our gratitude to the contributors for their effort to review the current status of neuroimmunomodulation in aging and consider future directions for research. We also thank the editor of Neuroimmunomodulation for supporting this special issue and for helping to see it to completion. The reviews and original papers presented here serve to remind us how much has already been accomplished in the field of neuroimmunomodulation of aging, and seem a useful foundation on which further research can be built. 